\title{
An Enhanced Stabilizing Strategy for Switched Nonlinear Systems*
}

\author{
Faiçal HAMIDI ${ }^{1}$, Houssem JERBI ${ }^{2 *}$, Severus Constantin OLTEANU ${ }^{3}$, Dumitru POPESCU \\ ${ }^{1}$ University of Gabès, Laboratory "Modelisation, Analyse et Commande des Systemes", LR16ES22, Gabès, Tunisia \\ faical.hmidi@isimg.tn \\ ${ }^{2}$ University of Ha'il, College of Engineering - Department of Industrial Engineering KSA \\ h.jerbi@uoh.edu.sa (*Corresponding author) \\ ${ }^{3}$ University Politehnica of Bucharest, Faculty of Automatic Control and Computer Science, Bucharest, Romania, \\ dumitru.popescu@acse.pub.ro
}

\begin{abstract}
This study examines a computational technique used to design a novel feedback control law based on an expansion strategy of the Attraction Domain (DA) for a class of nonlinear switched systems. It is supposed that the state space is distributed into numerous regions without any intersections and modelled by polynomial inequalities. The main concept involves the maximization of the DA for local subsystems surrounding particular operational points. It was demonstrated that the DA can be ascertained by joining a Genetic Algorithmic method (GA) as an enhanced optimisation approach with the LMI method for a specified Lyapunov function. The feedback controller can then be constructed in order to ensure a global stability by using the Multiple Lyapunov function sets via switching signals. The effectiveness of this evolved strategy is eventually confirmed via a simulation examination by means of the benchmark Van der Pol oscillator.
\end{abstract}

Keywords: Nonlinear switched models, Multiple Lyapunov function, Stability, LMI, Genetic Algorithms, Domain of Attraction (DA).

\section{Introduction}

Recently, an increased attention has been given to the examination of the class of nonlinear switched systems considered as a particular category of hybrid systems (Li \& Yang, 2019; Niu et al., 2017). These systems can be examined as remarkable as special types of hybrid system classes that are theoretically have advantageous in the application (Chesi, 2018; Luk \& Chesi, 2015). Switched systems can be described as dynamic systems that display numerous dynamic behaviors concurrently, in diverse portions of the systems under study. These are otherwise schemes that combine continuous or discrete timebased commands, jump occurrences, and logical commands (Pezeshki et al., 2018; Jerbi, 2017). These implementations involve a broad variety, including automotive, power grids, robotics, chemical processes, air traffic management networks and communication systems (Donkers

\footnotetext{
* This paper is an extended version of the paper called "Enlarging the Domain of Attraction in Nonlinear Polynomial Systems", published in the International Journal of Computers Communications and Control, $8(4), 538-547$. In the current paper, a feedback controller has been constructed to ensure a global stability for a class of hybrid systems by using the multiple Lyapunov function sets via switching signals. The control strategy is detailed and a synthesized algorithm is recommended. The effectiveness of this evolved strategy is eventually confirmed via a simulation examination. A numerical simulation analysis is carried out proving the satisfactory performances of the developed control scheme.
}

et al., 2011; Charfeddine et al., 2011). Stability analysis and the stabilization of nonlinear switched system types represent a problem addressed within many studies (Hamidi et al., 2009; Kermani \& Sakly, 2015; Pang \& Zhao, 2017). In the analysis of the stability of switched systems, certain outcomes have been achieved by means of a regular Lyapunov function, several Lyapunov function sets (Hamidi et al. (2011) and the idea of dwell time (Chesi \& Colaneri, 2017). As a consequence, drawn from this, it is notable that in the analysis of stabilities, numerous approaches have been recommended for linear switched systems (Liberzon, 2003; Zhang, 2014). Nevertheless, not many approaches have been recommended for nonlinear switched systems.

In this study, a novel nonlinear switched system stabilization strategy will be developed. The strategy is derived from the enlargement of an Attraction Domain for a localized subsystem (Hamidi et al., 2013; Jerbi et al., 2014). The design problem of specifying a subsystem relies on the necessary performance and control law objective considerations. The objective of the latest research is to develop precise techniques in order to enable the maximization of the DA, utilizing the genetic algorithmic (GA) methods as an enhanced optimization approach and also the quasi-convex methods entailing linear matrix inequalities (LMI) (Hamidi et al., 2013; Jerbi et al., 2014). The considered parametric optimization 
strategy manages the Lyapunov function (LF) and the controller input parameter set concurrently. The major purpose of this work is to develop a novel technique for facilitating the stability of a category of nonlinear switched systems. In particular, the objective of this paper is to extend the strategy synthesized in (Hamidi et al., 2013) and to make a full use of the obtained results to achieve the stabilization objective for a class of switched system. Generalized expressions will be given to describe the global stability of nonlinear switched systems.

This study is structured as follows: In section 2, the class of the examined systems is defined and the strategy for expanding the DA is presented. The control law hypothesis formulation is given in this section as well. In section 3 , the stability problem of the switched nonlinear systems where the subsystems exhibit stability will be introduced. Primary outcomes are illustrated in this section, where the designed conceptual algorithmic method is recommended. Section 4, is dedicated for an assessment of numerical simulations expanded towards validating the recommended strategy. Lastly, section 5 offers the conclusion and prospects for other areas in a future research.

\section{Problem Formulation}

\subsection{System Description}

Consider the nonlinear switched system defined by:

$\left\{\begin{array}{l}\dot{x}=f_{\sigma}(x)+g_{\sigma}(x) u_{\sigma}(x) \\ y=h_{\sigma}(x)\end{array}, \sigma=\{1,2, \ldots, N\}\right.$

wherein $x \in \mathfrak{R}^{n}$ represents the vectors of continuous state variables, $u_{\sigma} \in \mathfrak{R}^{p}$ denotes the control input vectors, and $y \in \mathfrak{R}^{q}$ denotes the quantifiable outputs. The index $\sigma$ denotes the discrete states sets assuming finite values. The vector function sets $f_{\sigma}(x), g_{\sigma}(x)$ and $h_{\sigma}(x)$ express the polynomial functions defining the mode $\sigma$ and verifying $f_{\sigma}(0)=0, g_{\sigma}(0)=0$ and $h_{\sigma}(0)$. The equilibrium point of interest, stable even for non-zero control input is the origin. Let $D_{\sigma} \subseteq \mathfrak{R}^{n}$ denotes the region of $\mathfrak{R}^{n}$ for piecewise non-linear system (1) expressed by:

$D_{\sigma}=\left\{x \in \mathfrak{R}^{n}: z_{\sigma}(x) \geq 0\right\}$

where $z_{\sigma}(x): \Re^{n} \rightarrow \mathfrak{R}^{n}$ is considered as a polynomial vector function. The studied switched systems are assumed to satisfy the following constraints:
1. $D_{\sigma} \cap D_{j}=\varnothing$ for all $\sigma \neq j$ and $\bigcup_{\sigma} D_{\sigma}=\Re^{n}$ such that the defined systems (1) are deterministic;

2. A step variation must occur before the path in mode $\sigma$ discards the matching continuous time state space $D_{\sigma}$ so that the nonlinear switched systems (1) do not engender the blocking behavior. This means that the switching law must be performed for each subsystem in its own definition domain where the switching occurs only when the state trajectory leaves region $D_{\sigma} 1$ or leaves region $D_{\sigma} 2$.

3. There is only a finite number of shifts in a finite time (i.e. the class of systems defined in model (1) does not show Zeno dynamics).

4. Sliding modes on the switching area do not take place.

\subsection{Control law Synthesis}

Control objectives involve the regulation of states of switched nonlinear systems towards the required equilibrium points with randomized switching in order to asymptotically stabilize their schemes. In every subsystem, the controller input is presumed to be expressed as a polynomial function:

$u_{\sigma}(y)=U_{\sigma} \phi(y)$

wherein $\phi(y) \in \mathfrak{R}^{r}$ is a provided polynomial for each sub-system's output $y$.

Consequently, the control law can be expressed using Kronecker vector notation as follows:

$u_{\sigma}(y)=\sum_{i=1}^{l} U_{\sigma, i} y^{[i]}$

wherein $l$ is a truncation order and $U_{\sigma, i} \in R^{p \times r}$ is a coefficients matrix belonging to the interval matrix defined by the set:

$\mathbf{M}=\left\{\begin{array}{l}U_{\sigma, i}=\left[U_{\sigma, 1} U_{\sigma, 2} \cdots U_{\sigma, l}\right] \text { where } \\ U_{\sigma, i}^{\min } \leq U_{\sigma, i} \leq U_{\sigma, i}^{\max } \text { for } \\ \sigma=1, \ldots, N \text { and } i=1, \ldots, l\end{array}\right\}$

For sake of clarity, the expression of the control law described by (3) developed under the polynomial form for $y=h_{\sigma}(x)=x$ and $l=3$, can be expressed as follows:

$u_{\sigma}(y)=U_{\sigma, 1} y^{[1]}+U_{\sigma, 2} y^{[2]}+U_{\sigma, 3} y^{[3]}$ 
where:

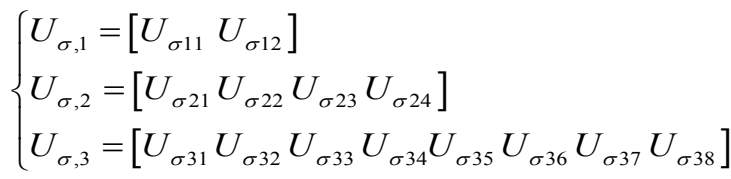

\subsection{Description of the LMI method}

Let us consider, for every subsystem described by (1), a positive definite function $V_{\sigma}(x) \in \mathfrak{R}$ which is positive definite, radially unbound and continually differentiable. The bounded sets for all the subsystems are provided in the following equation:

$\Omega_{\sigma}\left(c_{\sigma}\right)=\left\{x \in \mathfrak{R}^{n} \mid V_{\sigma}(x) \leq c_{\sigma}\right\}$

which approximates the domain of attraction if $\Omega_{\sigma}\left(c_{\sigma}\right) \subset D_{\sigma}$ wherein $D_{\sigma}=\left\{x \in \Re^{n} / \dot{V}_{\sigma}\left(x, U_{\sigma}\right)<0\right\} \cup\{0\}$ . The time-based derivative of $V_{\sigma}(x)$, down the system trajectory (1) is provided by:

$$
\begin{aligned}
\dot{V}_{\sigma}\left(x, U_{\sigma}\right) & =\frac{\partial V_{\sigma}(x)}{\partial x}\left(f_{\sigma}(x)+g_{\sigma}(x) U_{\sigma} \phi\left(h_{\sigma}(x)\right)\right) \\
& =\frac{\partial V_{\sigma}(x)}{\partial x} f_{\sigma}(x)+\frac{\partial V_{\sigma}(x)}{\partial x} g_{\sigma}(x) U_{\sigma} \phi\left(h_{\sigma}(x)\right) \\
& =L_{f_{\sigma}} V_{\sigma}(x)+L_{g_{\sigma}} V_{\sigma}(x) U_{\sigma} \phi\left(h_{\sigma}(x)\right)
\end{aligned}
$$

$L_{f_{\sigma}} V_{\sigma}(x)$, " $L_{g_{\sigma}} V_{\sigma}(x)$ respectively" denotes the Lie derivative of $V_{\sigma}(x) \in \mathfrak{R}$, along the vector $f_{\sigma}(x)$ " $g_{\sigma}(x)$ respectively". As follows, it is denoted with:

$$
L_{\left(g_{\sigma}, U_{\sigma}\right)} V_{\sigma}(x)=L_{g_{\sigma}} V_{\sigma}(x) U_{\sigma} \phi\left(h_{\sigma}(x)\right) \text {. }
$$

The greatest approximation of the attraction domain is $\Omega_{\sigma}\left(c_{\sigma}^{*}\left(U_{\sigma}\right)\right)$ and

$$
\left\{\begin{array}{l}
c_{\sigma}^{*}\left(U_{\sigma}\right)=\inf _{x \in R^{n}} V_{\sigma}(x) \\
\dot{V}_{\sigma}\left(x, U_{\sigma}\right)=0
\end{array}\right.
$$

resulting in optimal values for $c_{\sigma}^{*}\left(U_{\sigma}\right)$

$$
c_{\sigma}^{*}=\sup _{U_{\sigma} \in \mathcal{U}} c_{\sigma}^{*}\left(U_{\sigma}\right)
$$

It was demonstrated in (Hamidi et al., 2013) that for all the specified $c_{\sigma} \in \mathfrak{R}, c_{\sigma} \leq c_{\sigma}^{*}$ if $U_{\sigma}$ and positive definite function $s_{\sigma}(x)$ are obtained wherein:

$\dot{V}_{\sigma}\left(x, U_{\sigma}\right)+\left(c_{\sigma}-V_{\sigma}(x)\right) s_{\sigma}(x)<0$

then the polynomial degrees for $V_{\sigma}(x)$ and $\dot{V}_{\sigma}\left(x, U_{\sigma}\right)$, " $2 \delta_{V}$ and $\delta_{L}$, respectively. When selecting $s_{\sigma}(x)$ degree as $2 \delta_{s}$ for

$\delta_{s} \geq \frac{\delta_{L}}{2}-\delta_{V}$ therefore, the polynomial degree of:

$$
t_{\sigma}\left(x, U_{\sigma}, c_{\sigma}, s_{\sigma}(x)\right) \text { is }=\dot{V}_{\sigma}\left(x, U_{\sigma}\right)+\left(c_{\sigma}-V_{\sigma}(x)\right) s_{\sigma}(x)
$$

is equal to $2 \delta_{t}$ and $\delta_{t}=\delta_{s}+\delta_{V}$. For this study, square matrix representations (SMR) and complete-square matrix representations (CSMR) of polynomial expressions (Hamidi et al., 2013) are utilized so as to obtain suitable optimizable problems. These CSMR stipulate every possible representation of the polynomials under the quadratic forms.

The CSMR of:

$$
\begin{aligned}
& t_{\sigma}\left(x, U_{\sigma}, c_{\sigma}, s_{\sigma}(x)\right)= \\
& \quad x^{\left\{\delta_{t}\right\}^{\prime}}\left(D_{f_{\sigma}}(\alpha)+D_{g_{\sigma}}\left(U_{\sigma}\right)+c_{\sigma} W_{1}\left(S_{\sigma}\right)-W_{2}\left(S_{\sigma}\right)\right) x^{\left\langle\delta_{t}\right\}^{\prime}}
\end{aligned}
$$

is provided by:

$T_{\sigma}\left(\alpha, U_{\sigma}, c_{\sigma}, S_{\sigma}\right)=D_{f_{\sigma}}(\alpha)+D_{g_{\sigma}}\left(U_{\sigma}\right)+c_{\sigma} W_{1}\left(S_{\sigma}\right)-W_{2}\left(S_{\sigma}\right)$

Wherein $D_{f_{\sigma}}(\alpha)$ denotes the CSMR of $L_{f_{\sigma}} V_{\sigma}(x), D_{g_{\sigma}}\left(U_{\sigma}\right)$ denotes the SMR of $L_{\left(g_{\sigma}, U_{\sigma}\right)} V_{\sigma}(x), W_{1}\left(S_{\sigma}\right)$ and $W_{2}\left(S_{\sigma}\right)$ represent the SMR of $s_{\sigma}(x)$, and $V_{\sigma}(x) s_{\sigma}(x)$.

The conditionalities (11) and (17) indicates that if:

$\left\{\begin{array}{l}\hat{c}_{\sigma}^{*}=\sup _{U_{\sigma} \in \mathcal{U}, \alpha, S_{\sigma}>0} c_{\sigma} \\ T_{\sigma}\left(\alpha, U_{\sigma}, c_{\sigma}, S_{\sigma}\right)<0\end{array}\right.$

then $\hat{c}_{\sigma}^{*} \leq c_{\sigma}^{*}$.

The above description results in a non-convex problem (as $c_{\sigma}$ multiplies every parameter of $S_{\sigma}$ in $\left.T_{\sigma}\left(\alpha, U_{\sigma}, c_{\sigma}, S_{\sigma}\right)\right)$. The preceding hypotheses re-express the studied problem as a generalized eigenvalue type (GEVP) enabling the overcoming of the constraint (Hamidi et al., 2013).

Theorem 1 see (Hamidi et al. 2013) Lower bound $\hat{c}_{\sigma}^{*}$ as provided by

$\hat{c}_{\sigma}^{*}=\frac{-\lambda_{\sigma}^{*}\left(U_{\sigma}\right)}{1+\mu \lambda_{\sigma}^{*}\left(U_{\sigma}\right)}$

wherein $\lambda_{\sigma}^{*}\left(U_{\sigma}\right)$ the solution for GEVP is as follows $\lambda_{\sigma}^{*}\left(U_{\sigma}\right)=\inf _{U_{\sigma} \in u, \alpha, S>0, \lambda_{\sigma}} \lambda_{\sigma}$

s. $t\left\{\begin{array}{l}1+\mu \lambda_{\sigma}>0 \\ U_{\sigma} \in M \\ S_{\sigma}>0 \\ \lambda_{\sigma} W_{1}\left(S_{\sigma}\right)>D_{f_{\sigma}}(\alpha)+D_{g_{\sigma}}\left(U_{\sigma}\right)-W_{2}\left(S_{\sigma}\right)\end{array}\right.$ 
wherein $\mu$ is an arbitrary positive scalar, and:

$W_{1}\left(S_{\sigma}\right)=K^{T}\left(\left[\begin{array}{cc}1 & 0 \\ 0 & \mu V_{\sigma}\end{array}\right] \otimes S_{\sigma}\right) K$

wherein $\otimes$ denotes the Kronecker product and one has:

$\left[\begin{array}{c}1 \\ x^{\left\{\delta_{v}\right\}}\end{array}\right] \otimes x^{\left\{\delta_{s}\right\}}=K x^{\left\{\delta_{m}\right\}}$

wherein:

$x^{\left\{\delta_{t}\right\}} \in \mathfrak{R}^{\varsigma\left(n, \delta_{t}\right)}, \alpha \in \mathfrak{R}^{\tau\left(n, \delta_{t}\right)}$,

$x^{\left\{\delta_{V}\right\}} \in \mathfrak{R}^{\varsigma\left(n, \delta_{V}\right)}, x^{\left\{\delta_{s}\right\}} \in \mathfrak{R}^{\varsigma\left(n, \delta_{s}\right)}$

$K \in \mathfrak{R}^{\varsigma\left(n, \delta_{S}\right)\left(\varsigma\left(n, \delta_{V}\right)+1\right) \times \varsigma\left(n, \delta_{t}\right)}$

including $\varsigma\left(n, \delta_{m}\right)$ and ${ }^{\tau\left(n, \delta_{m}\right)}$ are provided by

$\varsigma\left(n, \delta_{t}\right)=\frac{\left(n+\delta_{t}\right) !}{n ! \delta_{t} !}-1$

$\tau\left(n, \delta_{t}\right)=\frac{1}{2} \varsigma\left(n, \delta_{t}\right)\left(\varsigma\left(n, \delta_{t}\right)+1\right)-\varsigma\left(n, 2 \delta_{t}\right)+n$

\section{Recommended Strategy}

\subsection{Stability Problem Analysis}

For the purpose of introducing the stability problem for the class of the studied systems, the oscillating element of the hybrid Van der Pol system (Rozgonyi et al., 2010; Liu et al., 2017) ) will be examined. This scheme may comprise the hybrid attribute whenever the values used in constructing the circuit are indeterminate. These results in a modelled state for indeterminate coefficients that can be regarded as a hybrid scheme described by numerous subsystems. For this instance, "the hybrid Van der Pol system is described by using dual dissimilar models with continuous dynamics provided by:

\section{Subsystem 1}

$\dot{x}=f_{1}(x)=\left\{\begin{array}{l}\dot{x}_{1}=-1.2 x_{2} \\ \dot{x}_{2}=2 x_{1}-0.65 x_{2}+1.1 x_{1}^{2} x_{2}\end{array}\right.$

\section{Subsystem 2}

$\dot{x}=f_{2}(x)=\left\{\begin{array}{l}\dot{x}_{1}=-1.2 x_{2} \\ \dot{x}_{2}=1.86 x_{1}-1.01 x_{2}+2.1 x_{1}^{2} x_{2}\end{array}\right.$

To assess the stabilities of the subsystems (23) and (24), the linearized systems are examined at their origin, corresponding to these matrices as follows:
$A_{1}=\left(\begin{array}{cc}0 & -1.2 \\ 2 & -0.65\end{array}\right) ; \quad A_{2}=\left(\begin{array}{cc}0 & -1.2 \\ 1.86 & -1.01\end{array}\right)$

having dual complex conjugate poles corresponding to

$(-0.325+1.5147 i),(-0.325-1.5147 i)$

and $(-0.505+1.406 i),(-0.505-1.406 i)$

with negative real parts. This demonstrates that both subsystems feature global asymptotic stability. Diverse dynamics are described in their domains in order to fully accomplish the hybrid Van der Pol system.

$D_{1}=\left\{x_{1}>0.6 \wedge\left(0.36-x_{1}^{2}\right) x_{2}>0.14 x_{1}\right\}$

$D_{2}=\mathfrak{R} \backslash X_{1}$

Switching signals are illustrated in Figure 1.

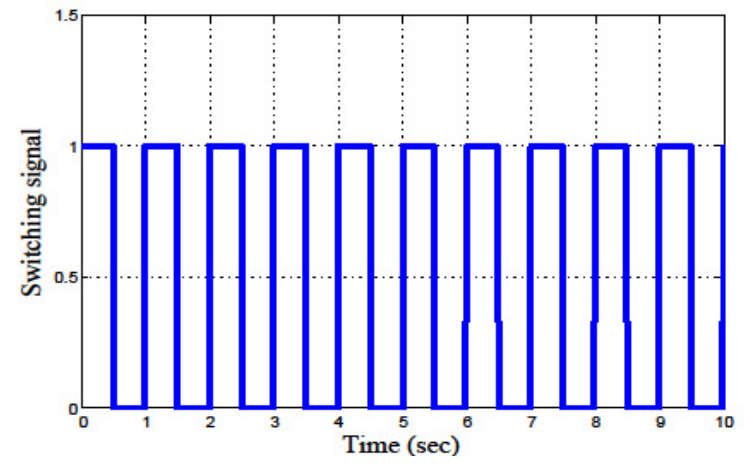

Figure 1. Switching signals

As shown in Figure 2, the asymptotically stable region contains the equilibrium point $(0,0)$. Nevertheless, from the initialised condition $(0.69,1.9)$ and with the randomised switching signals, the developing state dynamics of the switched systems deviate since, at the final commutation at time $t_{c_{1}}=3.59 \mathrm{~s}$, these states lie beyond the asymptotically stable region.

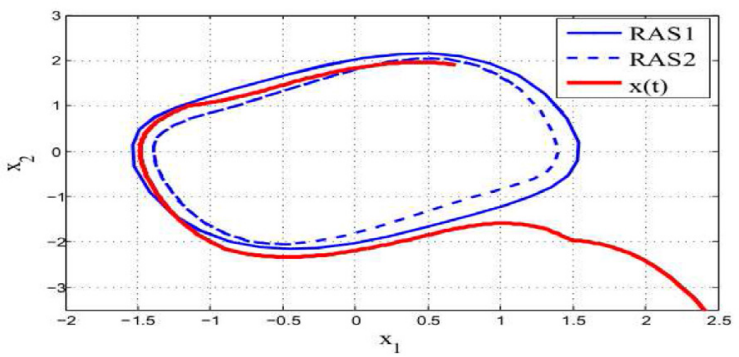

Figure 2. Regions of asymptotic stability RAS1 (solid blue lines), RAS2 (dashed) and $x(t)$ trajectories of switched systems (solid red lines).

In accordance with such simulations of switched systems, it can be determined that switching 
among stable subsystems does not inevitably result in a stable dynamic scheme. Therefore, the stabilities of switched systems rely not just on the continuously dynamic natures of these subsystems but also on exploitation of the switching approach.

\subsection{Description of GA Method}

In what follows, the primary outcomes that have been achieved in the current study will be introduced. Hence, the Reverse Trajectory Method (RTM) presented in (Hamidi et al. 2013) will be applied to approximate the Region of Asymptotic Stability (RAS) via a reversal of the systemic trajectory flow. the primary aim of this work is to expand the RAS that results from the technique defined in (Hamidi et al. 2013), enabling the determination of the analytical formulations of the maximum DA within the RAS. To simplify the proposed approach, two subsystems have been assumed. The results can be generalized for several subsystems. Thereby the Lyapunov function of the quadratic form is the following:

$V_{1}(x)=x^{T} P x$

$V_{2}(x)=x^{T} Q x$

wherein $P=P^{T}>0, Q=Q^{T}>0$ for maintaining simplicity, it is assumed that $P, Q \in \mathfrak{R}^{2}$, where $P=\left[\begin{array}{ll}p_{11} & p_{12} \\ p_{12} & p_{22}\end{array}\right]$ and $Q=\left[\begin{array}{ll}q_{11} & q_{12} \\ q_{12} & q_{22}\end{array}\right]$ such that

$V_{1}(x)=p_{11} x_{1}^{2}+2 p_{12} x_{1} x_{2}+p_{22} x_{2}^{2}$

$V_{2}(x)=q_{11} x_{1}^{2}+2 q_{12} x_{1} x_{2}+q_{22} x_{2}^{2}$

The resulting expressions can be generalised for larger-sized matrices. Through the utilisation of the Lyapunov functions, the RAS can be formulated as being determined by the RTM see (Hamidi et al., 2011), in the form of ellipsoids in the $\left(x_{1}, x_{2}\right)$ plane. The procedure involves a parametric estimation of $p_{\sigma j}, q_{\sigma j} ;(\sigma=1,2 ; j=1,2)$ and $u_{\sigma j},(\sigma=1,2 ; j=1,2)$ through genetic algorithmic methods see (Moussa et al. 2018, Serban et al., 2016). By joining such algorithmic methods through LMI optimisations, the largest ellipsoid bounded within the RAS can be determined. The set of candidate solution, $p_{\sigma j}, q_{\sigma j} ;(\sigma=1,2 ; j=1,2)$ and $u_{\sigma j},(\sigma=1,2 ; j=1,2)$ is randomly selected and can be assumed to be represented by discrete elements. Every variable can be examined as a genetic factor, with various phases of this genetic algorithmic method expressed via the dissimilar operation of GA see (Moussa et al. 2018; Serban et al., 2016).

\section{Remarks:}

(i) For this study, a GA is utilised in order to approximating the parameters $p_{\sigma j}, q_{\sigma j},(\sigma=1,2 ; j=1,2)$ and $u_{\sigma j},(\sigma=1,2 ; j=1,2)$ wherein the limit is satisfied by:

$$
\begin{aligned}
& p_{11}>0, p_{22}>0, p_{11} \cdot p_{22}>\left(p_{12}\right)^{2} \\
& q_{11}>0, q_{22}>0, q_{11} \cdot q_{22}>\left(q_{12}\right)^{2}
\end{aligned}
$$

(ii) Every reiteration of the GA optimisation sequence leads to new parametric values, $p_{\sigma j}, q_{\sigma j},(\sigma=1,2 ; j=1,2)$, $u_{\sigma j},(\sigma=1,2 ; j=1,2)$ and $\hat{c}_{\sigma}^{*}$ that represent a solution set of the LMI with respect to this parameter set. GA approach results in the most appropriate parameter set,

$p_{\sigma j}, q_{\sigma j},(\sigma=1,2 ; j=1,2)$ and $u_{\sigma j},(\sigma=1,2 ; j=1,2)$ that can be utilised for calculating of the superior solution $\hat{c}_{\sigma}^{*}$ (described in (17)), representing the resolution of LMI (18).

The variable sets

$\left\{p_{\sigma j}, q_{\sigma j} ;(\sigma=1,2 ; j=1,2)\right\}$ and $\left\{u_{\sigma j},(\sigma=1,2 ; j=1,2)\right\}$ are encoded into genetic candidates. Fitness values are provided by the maximal value of $\hat{c}_{\sigma}^{*}$ for which there are possible solutions to the optimisation of the LMI. Global optimisations of the variable set

$p_{\sigma j}, q_{\sigma j}(\sigma=1,2 ; j=1,2) \quad u_{\sigma j},(\sigma=1,2 ; j=1,2)$ are conducted using the genetic operators (recombination, mutation, and selection see (Moussa et al., 2018; Serban et al., 2016).

(iii) The Expansions of attraction domain are associated with the optimisation criteria of ellipses, as described in the following expression:

$\Omega_{\sigma}\left(\hat{c}_{\sigma}^{*}\right)=\left\{x \in \mathfrak{R}^{n} \mid x^{T} P x \leq \hat{c}_{\sigma}^{*}\right\}$

Volumes are expressed by:

$$
\operatorname{Vol}\left(\Omega_{\sigma}\right)=\frac{\hat{c}_{\sigma}^{*}}{\sqrt{\operatorname{det}(P)}}
$$




\subsection{Synthesis of the Algorithmic Method}

The primary pseudo code is required for executing the synthesised algorithmic method, where accordingly, the optimal values for the parametric radii of domains of attraction, is expressed as:

Algorithm. Computing the largest DA estimates for each subsystem

Input:

- A Quadratic Lyapunov function for each subsystem,

$$
\begin{aligned}
& V_{1}(x)=p_{11} x_{1}^{2}+2 p_{12} x_{1} x_{2}+p_{22} x_{2}^{2} \\
& V_{2}(x)=q_{11} x_{1}^{2}+2 q_{12} x_{1} x_{2}+q_{22} x_{2}^{2}
\end{aligned}
$$

- A controller law

$$
u_{\sigma}=\sum_{j=1}^{q} U_{\sigma j} x^{[j]} ; \sigma=1,2
$$

Output: a larger domain of attraction and a corresponding controller law for each subsystem.

Set $p_{\sigma j}, q_{\sigma j}, u_{\sigma j}(\sigma=1,2 ; j=1,2)$ as candidate genetic factors

\section{whereas}

$p_{11}>0, p_{22}>0, p_{11} \cdot p_{22}>\left(p_{12}\right)^{2}$

$q_{11}>0, q_{22}>0, q_{11} \cdot q_{22}>\left(q_{12}\right)^{2}$

$k=1$;

while $k<=+\infty$ do

solve the LMI optimisation (17)-(18);

if $V_{\sigma, k+1}(x)$ exists then

$$
\begin{aligned}
& D_{\sigma, k+1}\left\{x: V_{\sigma, k+1}(x) \leq c_{\sigma, k+1}\right\} \\
& u_{\sigma, k+1}=\sum_{j=1}^{q} U_{\sigma, k+1} x^{[j]}, k:=k+1
\end{aligned}
$$

end if

end while

return

$$
\begin{aligned}
& D_{\sigma, k}\left\{x: V_{\sigma, k}(x) \leq c_{\sigma, k}\right\} \\
& u_{\sigma, k}=\sum_{j=1}^{q} U_{\sigma, k} x^{[j]}
\end{aligned}
$$

The flowchart provided in the following Figure 3 depicts the design methodology, the asymptotically stable region has been maximised and the stabilisation for the switched nonlinear system has been validates, where appropriate. "LF denotes Lyapunov function".

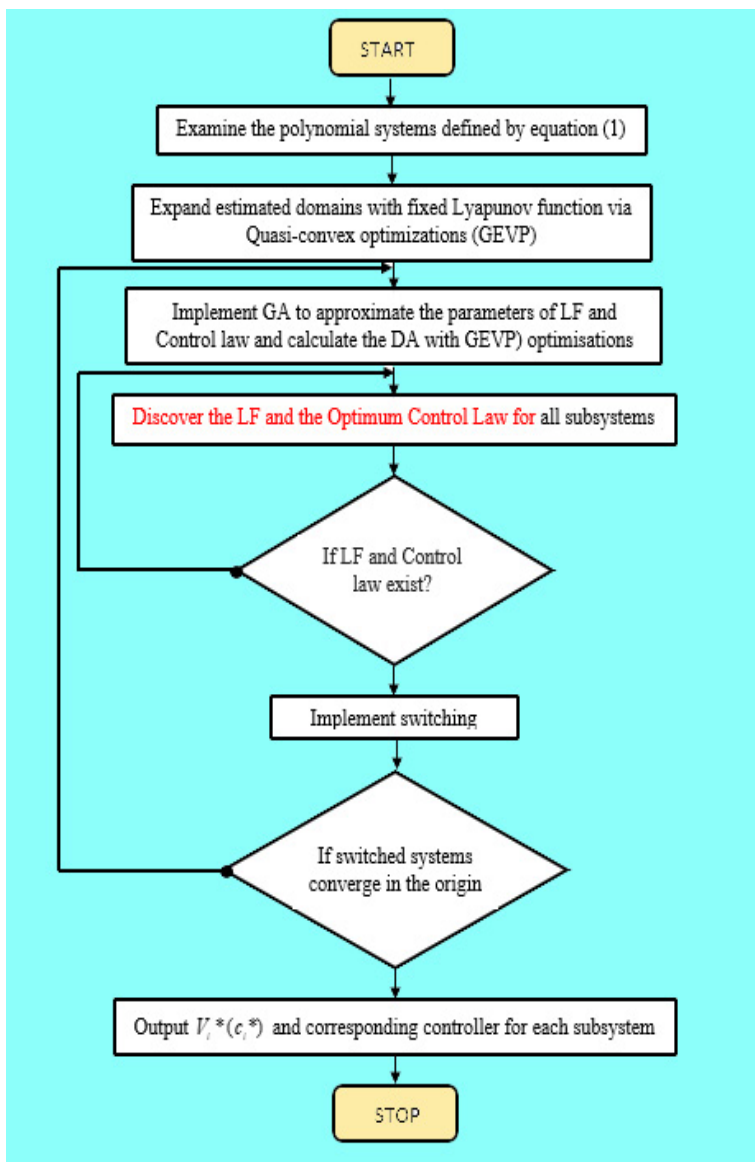

Figure 3. Flowchart of the design methodology

\section{Study of an Illustrative Example}

\subsection{System Modeling}

To explain the primary operation of the recommended approach, this switched Van der Pol system is examined as follows see (Rozgonyi et al., 2010):

\section{Subsystem 1:}

$$
\dot{x}=f_{1}(x)=\left\{\begin{array}{l}
\dot{x}_{1}=-1.2 x_{2} \\
\dot{x}_{2}=2 x_{1}-0.65 x_{2}+1.1 x_{1}^{2} x_{2}+u_{1}(x)
\end{array}\right.
$$

\section{Subsystem 2:}

$$
\dot{x}=f_{2}(x)=\left\{\begin{array}{l}
\dot{x}_{1}=-1.2 x_{2} \\
\dot{x}_{2}=1.86 x_{1}-1.01 x_{2}+2.1 x_{1}^{2} x_{2}+u_{2}(x)
\end{array}\right.
$$

Formulations of the recommended controllers (4) are determined for $y_{1}=\left[x_{1}, x_{2}\right]^{T}$ and $y_{2}=\left[x_{1}, x_{2}\right]^{T}$ by $u_{1}(x)=U_{11} x_{1}+U_{12} x_{2}$ and $u_{2}(x)=U_{21} x_{1}+U_{22} x_{2}$ as with

$$
\begin{aligned}
& U_{1}=\left\{\left[U_{11}, U_{12}\right]:-2 \leq U_{11}, U_{12} \leq 2\right\} \\
& U_{2}=\left\{\left[U_{21}, U_{22}\right]:-2 \leq U_{21}, U_{22} \leq 2\right\}
\end{aligned}
$$


As the aim of this study is to confirm the stabilities of hybrid Van der Pol models, these analytical processes have been initially employed in order to expend their domains of attraction as depicted in (Hamidi et al., 2013). For this purpose, the polynomial Lyapunov functions, describing every subsystem and truncating to the first order, have been applied as follows:

\section{- The first subsystem}

$V_{1}(x)=p_{11} x_{1}^{2}+2 p_{12} x_{1} x_{2}+p_{22} x_{2}^{2}$

Given that the degree $\delta_{L}$ of $\dot{V}_{1}(x, U)$ equals 4, through a setting of $\delta_{s}=1 \delta_{t}=2$ is obtained. Vectors $x^{\left\{\delta_{V}\right\}}, x^{\left\{\delta_{s}\right\}}$ and $x^{\left\{\delta_{t}\right\}}$ are designated as: $\quad x^{\left\{\delta_{V}\right\}}=x^{\left\{\delta_{s}\right\}}=\left[\begin{array}{ll}x_{1} & x_{2}\end{array}\right]^{T}$ and $x^{\left\{\delta_{t}\right\}}=\left[x_{1}, x_{2}, x_{1}^{2}, x_{1} x_{2}, x_{2}^{2}\right]^{T}$ implying:

$D_{f_{1}}\left(\alpha_{1,2,3}, \beta_{1,2,3}\right)=\left(\begin{array}{ccccc}4 p_{12} & \beta_{1} & 0 & \alpha_{1} & \alpha_{2} \\ \beta_{1} & \beta_{2} & -\alpha_{1} & -\alpha_{2} & 0 \\ 0 & -\alpha_{1} & 0 & 1.1 p_{12} & \alpha_{3} \\ \alpha_{1} & -\alpha_{2} & 1.1 p_{12} & \beta_{3} & 0 \\ \alpha_{2} & 0 & \alpha_{3} & 0 & 0\end{array}\right)$

$\beta_{1}=\left(2 p_{22}-1.2 p_{11}-0.65 p_{12}\right)$,

$\beta_{2}=\left(-2.4 p_{12}-1.3 p_{22}\right), \beta_{3}=2\left(1.1 p_{22}-\alpha_{3}\right)$

$D_{g_{1}}\left(a_{1,2,3}\right)=\left(\begin{array}{ccccc}a_{1} & a_{2} & 0 & 0 & 0 \\ a_{2} & a_{3} & 0 & 0 & 0 \\ 0 & 0 & 0 & 0 & 0 \\ 0 & 0 & 0 & 0 & 0 \\ 0 & 0 & 0 & 0 & 0\end{array}\right)$

$a_{1}=2 p_{12} U_{11}, a_{2}=\left(p_{22} U_{11}+p_{12} U_{12}\right), a_{3}=2 p_{22} U_{12}$.

$W_{1}\left(S_{\sigma, j=1,2}, k_{1, . .5},\right)=\left(\begin{array}{ccccc}s_{11} & s_{12} & 0 & 0 & 0 \\ s_{12} & s_{22} & 0 & 0 & 0 \\ 0 & 0 & k_{1} & k_{2} & 0 \\ 0 & 0 & k_{2} & k_{3} & k_{4} \\ 0 & 0 & 0 & k_{4} & k_{5}\end{array}\right)$

$k_{1}=\mu q_{11} s_{11}, k_{2}=\mu\left(q_{12} s_{11}+q_{11} s_{12}\right)$;

$k_{3}=\mu\left(q_{22} s_{I 1}+4 q_{12} s_{12}+q_{11} s_{22}\right)$;

$k_{4}=\mu\left(q_{12} s_{22}+q_{22} s_{12}\right), k_{5}=\mu q_{22} s_{22}$.

$W_{2}\left(r_{1, \ldots 5}\right)=\left(\begin{array}{ccccc}0 & 0 & 0 & 0 & 0 \\ 0 & 0 & 0 & 0 & 0 \\ 0 & 0 & r_{1} & r_{2} & 0 \\ 0 & 0 & r_{2} & r_{3} & r_{4} \\ 0 & 0 & 0 & r_{4} & r_{5}\end{array}\right)$

$r_{1}=p_{11} s_{11}, r_{2}=\left(p_{12} s_{11}+p_{11} s_{12}\right)$

$r_{3}=\left(p_{22} s_{11}+4 p_{12} s_{12}+p_{11} s_{22}\right)$;

$r_{4}=\left(p_{12} s_{22}+p_{22} s_{12}\right), r_{5}=p_{22} s_{22}$.
$S_{11,12,22}=\left(\begin{array}{ll}s_{11} & s_{12} \\ S_{12} & s_{22}\end{array}\right)$

\section{The second subsystem}

The Lyapunov function is described as follows:

$V_{2}(x)=q_{11} x_{1}^{2}+2 q_{12} x_{1} x_{2}+q_{22} x_{2}^{2}$

Given that the degree $\delta_{L}$ of $\dot{V}_{2}(x, U)$ is equal to 4 , by establishing $\delta_{s}=1$, and then $\delta_{t}=2$ is obtained. Vectors $x^{\left\{\delta_{V}\right\}}, x^{\left\{\delta_{s}\right\}}$ and $x^{\left\{\delta_{t}\right\}}$ are designated as: $\quad x^{\left\{\delta_{V}\right\}}=x^{\left\{\delta_{s}\right\}}=\left[\begin{array}{ll}x_{1} & x_{2}\end{array}\right]^{T}$, $x^{\left\{\delta_{t}\right\}}=\left[x_{1}, x_{2}, x_{1}^{2}, x_{1} x_{2}, x_{2}^{2}\right]^{T}$ implying:

$D_{f_{2}}\left(\alpha_{1,2,3}, e_{1,2,3}\right)=\left(\begin{array}{ccccc}3.72 q_{12} & e_{1} & 0 & \alpha_{1} & \alpha_{2} \\ e_{1} & e_{2} & -\alpha_{1} & -\alpha_{2} & 0 \\ 0 & -\alpha_{1} & 0 & 2.1 q_{12} & \alpha_{3} \\ \alpha_{1} & -\alpha_{2} & 2.1 q_{12} & e_{3} & 0 \\ \alpha_{2} & 0 & \alpha_{3} & 0 & 0\end{array}\right)$

$e_{1}=\left(1.86 q_{22}-1.2 q_{11}-1.01 q_{12}\right)$,

$e_{2}=\left(-2.4 q_{12}-2.02 q_{22}\right), e_{3}=2\left(2.1 q_{22}-\alpha_{3}\right)$.

$D_{g_{2}}\left(d_{1,2,3}\right)=\left(\begin{array}{ccccc}d_{1} & d_{2} & 0 & 0 & 0 \\ d_{2} & d_{3} & 0 & 0 & 0 \\ 0 & 0 & 0 & 0 & 0 \\ 0 & 0 & 0 & 0 & 0 \\ 0 & 0 & 0 & 0 & 0\end{array}\right)$

$d_{1}=2 q_{12} U_{21}, d_{2}=\left(q_{22} U_{21}+q_{12} U_{22}\right), d_{3}=2 q_{22} U_{22}$.

$W_{1}\left(m_{\sigma, j=1,2}, q_{1, \ldots .}\right)=\left(\begin{array}{ccccc}m_{11} & m_{12} & 0 & 0 & 0 \\ m_{12} & m_{22} & 0 & 0 & 0 \\ 0 & 0 & h_{1} & h_{2} & 0 \\ 0 & 0 & h_{2} & h_{3} & h_{4} \\ 0 & 0 & 0 & h_{4} & h_{5}\end{array}\right)$

$W_{2}\left(h_{1, \ldots 5}\right)=\frac{1}{\mu} \cdot\left(\begin{array}{ccccc}0 & 0 & 0 & 0 & 0 \\ 0 & 0 & 0 & 0 & 0 \\ 0 & 0 & h_{1} & h_{2} & 0 \\ 0 & 0 & h_{2} & h_{3} & h_{4} \\ 0 & 0 & 0 & h_{4} & h_{5}\end{array}\right)$

$h_{1}=\mu q_{11} m_{11}, h_{2}=\mu\left(q_{12} m_{11}+q_{11} m_{12}\right)$;

$h_{3}=\mu\left(q_{22} m_{11}+4 q_{12} m_{12}+q_{11} m_{22}\right)$;

$h_{4}=\mu\left(q_{12} m_{22}+q_{22} m_{12}\right), h_{5}=\mu q_{22} m_{22}$.

$M=\left(\begin{array}{ll}m_{11} & m_{12} \\ m_{12} & m_{22}\end{array}\right)$ 
The assessment of the DA maximization is continued by approximating the parameter sets, $\left(p_{11}, p_{12}, p_{22}\right)$ and $\left(q_{11}, q_{12}, q_{22}\right)$ of the corresponding Lyapunov functions $V_{1}(x)$ and $V_{2}(x)$, as well as $\left(U_{11}, U_{12}\right)$ , $\left(U_{21}, U_{22}\right)$ of the controller inputs $u_{1}(x)$ and $u_{2}(x)$. The parametric set, $p_{\sigma j}, q_{\sigma j} ; \sigma, j=1,2$ and $u_{\sigma j} ; \sigma, j=1,2$ should be encoded into the following 7-bit form:

$$
\begin{aligned}
& p_{\sigma j}=\left(p_{\sigma j}^{0}, p_{\sigma j}^{1}, p_{\sigma j}^{2}, p_{\sigma j}^{3}, p_{\sigma j}^{4}, p_{\sigma j}^{5}, p_{\sigma j}^{6}\right) ; \\
& q_{\sigma j}=\left(q_{\sigma j}^{0}, q_{\sigma j}^{1}, q_{\sigma j}^{2}, q_{\sigma j}^{3}, q_{\sigma j}^{4}, q_{\sigma j}^{5}, q_{\sigma j}^{6}\right) ; \\
& u_{\sigma j}=\left(u_{\sigma j}^{0}, u_{\sigma j}^{1}, u_{\sigma j}^{2}, u_{\sigma j}^{3}, u_{\sigma j}^{4}, u_{\sigma j}^{5}, u_{\sigma j}^{6}\right) .
\end{aligned}
$$

In order to be placed into the chromosomes characterised by the following parameter sets:

$\left\{\begin{array}{l}\text { Pop_size }=100 ; \quad \text { Crossover_rate }=0.65 ; \\ \text { Mutation }=0.1 ; \quad \text { Max_generations }=100 .\end{array}\right.$

Whereupon, the recommended technique has been implemented,

$V_{1}=1.597 x_{1}^{2}-0.598 x_{1} x_{2}+0.369 x_{2}^{2}=6.1423$

$V_{2}=1.6 x_{1}^{2}-1.82 x_{1} x_{2}+1.5 x_{2}^{2}=2.4916$

such that the inputs are provided by:

$u_{1}=1.4703 x_{1}-1.9996 x_{2}$

$u_{2}=-1.5738 x_{1}-1.9992 x_{2}$

The resulting values are presented in Figure 5 using $\mu=1 / 10$. It can be noted that the asymptotically stable regions and both DA of the Van der Pol hybrid models have been acquired through the Lyapunov functions $V_{1}(x)$ and $V_{2}(x)$, validating the asymptotic stability localised at the equilibrium point $(0,0)$. Furthermore, it should be noted that both the acquired RSA subsystems are strongly associated and include each other.

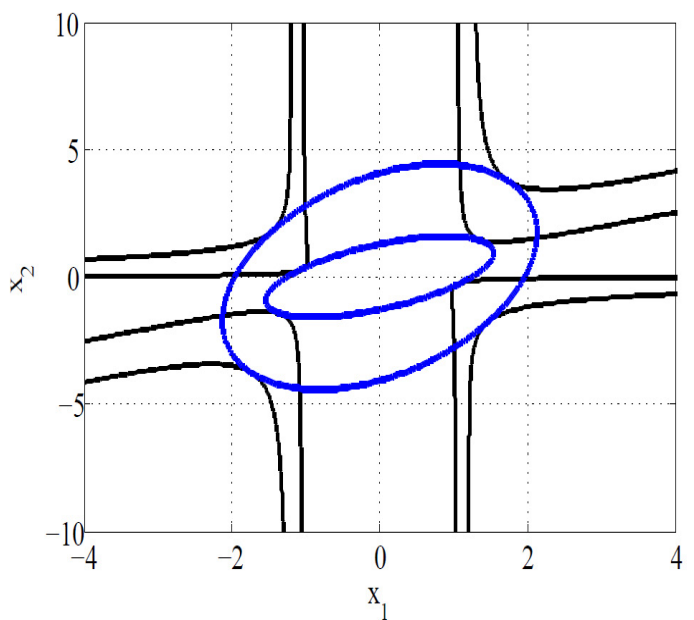

Figure 4. Ellipsoid shapes representing the Domains of Attraction for subsystems 1 and 2. Solid lines depict $\dot{V}_{1}(x, U)$ and $\dot{V}_{2}(x, U)$

\subsection{Results and Discussion}

The switching signals have been defined in Figure 1, with constructed trajectories switching between dual regions in square forms. Simulation outcomes expressing the dynamics of the switched non-linear schemes are provided in Figures 5-6. Figure 5 illustrates the state responses of switched non-linear systems for random initial states. Thus, it appears that the state trajectories asymptotically converge to their origin. Figure 6 depicts the sufficient behaviours of the state variables $x_{1}(t)$ and $x_{2}(t)$ of the switched non-linear system, even though at the beginning, the conditions have been established rather far from the origin. Apparently, the state variables converge at the origin with randomised switching signals, determining the asymptotic stabilities of the hybrid non-linear system.

A profile of the Lyapunov functions relevant to subsystems 1 and 2, for switching approaches designed in accordance with the stable condition of the switched systems is illustrated in Figure 7. It is fully demonstrated that this Lyapunov function validates the condition. Describing the system as it enters subsystem $i$, the value of the Lyapunov function linked with the subsystem has to be lower than the value obtained when the system previously left subsystem $i$. The controller signals depicted in Figure 8 describe a sufficiently dynamic development as it results in the immediately stabilisation of the switched non-linear system.

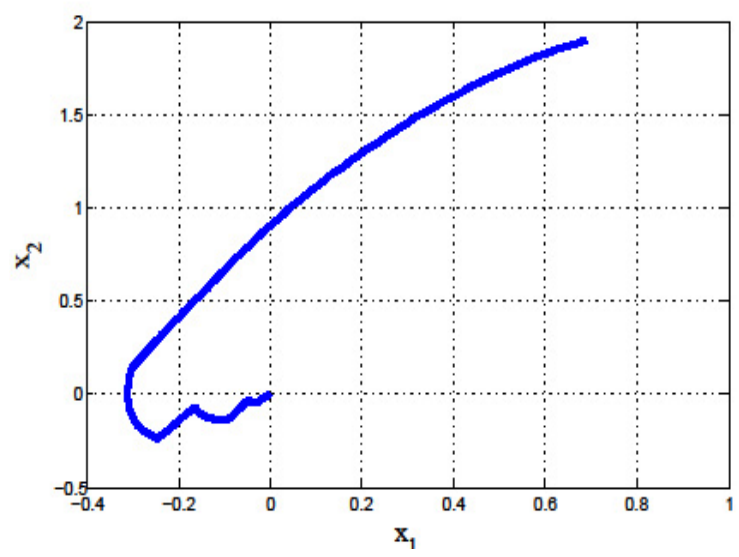

Figure 5. State trajectory of switched non-linear systems 


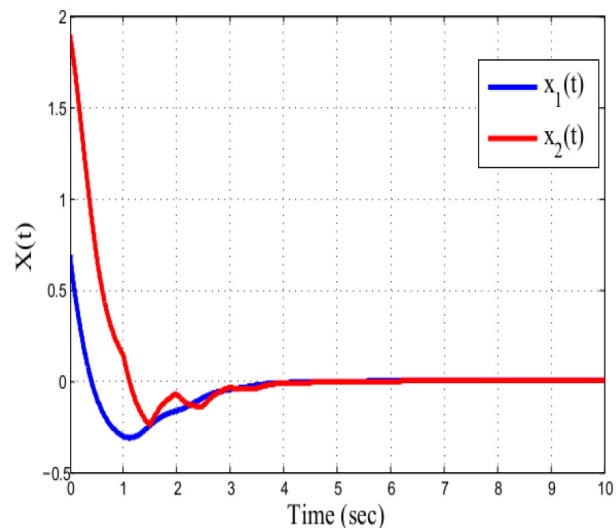

Figure 6. Development of the state trajectories of switched non-linear systems

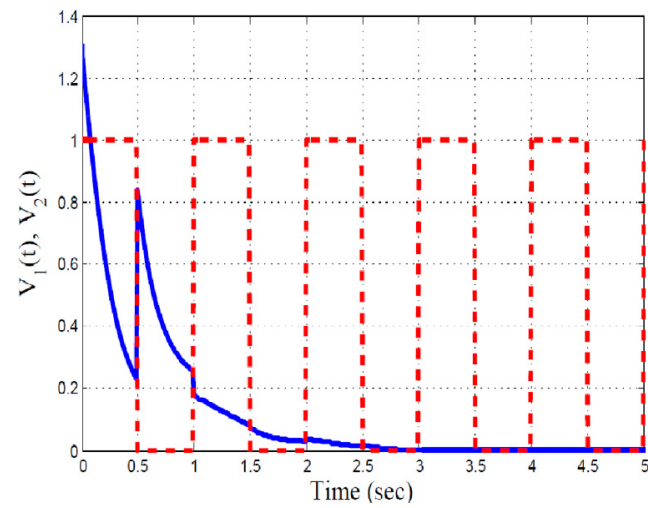

Figure 7. Development of the Lyapunov Function

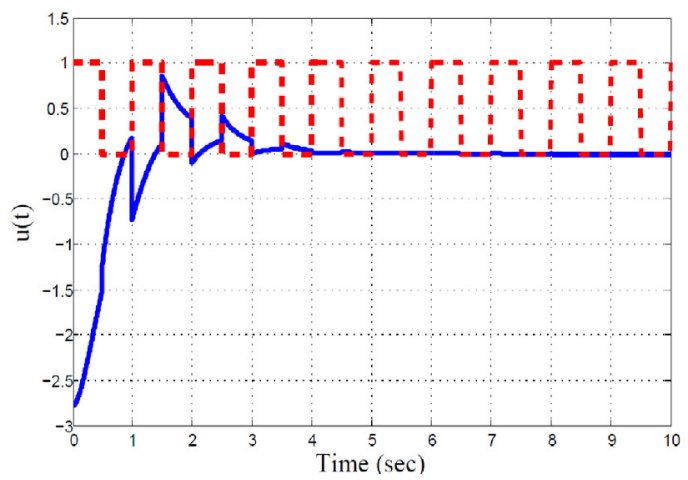

Figure 8. Development of the control law

\section{Conclusion}

The synthesis of a feedback control methodology which ensures an improved enlargement of the domain of attraction is the key concern analysed in this paper. The analysis has been primarily aimed at the class of the switched nonlinear polynomial systems wherein the dynamics is continuous on the boundary of the various regimes of the state space. The key contribution consists in establishing an unequivocal asymptotically stable region by exploiting the parameterised specific Lyapunov functions of the elementary sub-scheme. The gains of the nonlinear control variable as well as the parameters of Lyapunov function are calculated by merging the GA heuristic routine with a LMI-based method. A detailed flowchart has been prepared for offering a general approach in order to provide the stability for the class of system under consideration. The efficiency of the developed approach was verified by means of a simulation study of a hybrid Van der Pol system. Future research will focus on generalising this approach for the class of analytical non polynomial switched mechanisms.

\section{REFERENCES}

1. Charfeddine, M., Jouili, K., Jerbi, H. \& Benhadj Braiek, N. (2011). Output Tracking Control Design for Non-minimum Phase Systems: Application to the Ball and Beam Model, International Review of Automatic Control, 4(1), 47-55.

2. Chesi, G. \& Colaneri, P. (2017). Homogeneous Rational Lyapunov Functions for Performance Analysis of Switched Systems with Arbitrary Switching and Dwell Time Constraints, IEEE Transactions on Automatic Control, 62(10), 5124-5137.

3. Donkers, M., Heemels, W., Van de Wouw, N. \& Hetel, L. (2011). Stability Analysis of Networked Control Systems Using a Switched Linear Systems Approach, IEEE Transactions on Automatic control, 56(9), 2101-2115.

4. Hamidi, F. \& Jerbi, H. (2009). On the Estimation of a Maximal Lyapunov Function and Domain of Attraction Determination via a Genetic Algorithm. In $6^{\text {th }}$ International Multi-Conference on Systems, Signals and Devices (SSD) (pp.1-6).

5. Hamidi, F., Jerbi, H., Aggoune, W., Djemai, \& Abdekrim, M. N. (2011). Enlarging Region of Attraction via LMI-Based Approach and Genetic Algorithm. In International Conference on Communications, Computing and Control Applications (CCCA) (pp. 1-6).

6. Hamidi, F., Jerbi, H., Aggoune. W., Djemai, M. \& Abdelkrim, M. (2013). Enlarging the Domain of Attraction in Nonlinear Polynomial Systems, International Journal of Computers Communications and Control, $8(4), 538-547$. 
7. Jerbi, H., Ben Hadj Braiek, N. \& Bacha, B. (2014). A Method of Estimating the Domain of Attraction for Nonlinear Discrete-Time Systems, Arabian Journal of Science and. Engineering, 39(5), 3841-3849.

8. Jerbi, H. (2017). Estimations of the Domains of Attraction for Classes of Nonlinear Continuous Polynomial Systems, Arabian Journal of Science and Engineering, 42(7), 2829-2837.

9. Kermani, M. \& Sakly, A. (2015). Delay Independent Stability Criteria Under Arbitrary Switching of a Class of Switched Nonlinear Time-delay Systems, Advances in Difference Equations, 2015(1), Article number: 225. DOI: 10.1186/s13662-0150560-1

10. Li, C. \& Yang, X. (2019). Adaptive Neural Output Feedback Control for a Class of Switched Non-linear Systems with Unknown Backlash-like Hysteresis of the Actuator, Transactions of the Institute of Measurement and Control, 41(4), 900-910.

11. Liberzon, D. (2003). Switching in Systems and Control. Springer Science and Business Media, LLC, Birkhäuser.

12. Liu, L., Zhou, Q., Liang, H. \& Wang, L. (2017). Stability and Stabilization of Nonlinear Switched Systems Under Average Dwell Time, Applied Mathematics and Computation, 298, 77-94.

13. Luk, C. K. \& Chesi, G. (2015). Guaranteed Estimates of the Domain of Attraction for a Class of Hybrid Systems, International Journal of Robust and Nonlinear Control, 25(17), 3270-3285.
14. Moussa, M., Hmila, M. \& Douik, A. (2018). A Novel Face Recognition Approach Based on Genetic Algorithm Optimization, Studies in Informatics and Control, 27(1), 127-134. DOI: 10.24846/v27i1y201813

15. Niu, B., Karimi, H., Wang, H. \& Liu, Y., (2017). Adaptive Output-feedback Controller Design for Switched Nonlinear Stochastic Systems with a Modified Average Dwell-time Method, IEEE Transactions on Systems, Man, and Cybernetics: Systems, 47(7), 1371-1382.

16. Pang, H. \& Zhao, J. (2017). Adaptive Feedback Passivity-based Disturbance Attenuation for Switched Nonlinearly Parameterized Systems, Transactions of the Institute of Measurement and Control, 39(12), 1811-1820.

17. Pezeshki, S., Badamchizadeh, M., Ghiasi, A. \& Ghaemi, S. (2018). Stability Analysis and Robust Tracking Control for a Class of Switched Nonlinear Systems with Uncertain Input Delay, Transactions of the Institute of Measurement and Control, 41(7), 2053-2063.

18. Rozgonyi, S., Hangos, K. \& Szederknyi, G. (2010). Determining the Domain of Attraction of Hybrid Non-linear Systems Using Maximal Lyapunov Functions, Kybernetika, 46(1), 19-37.

19. Serban, C. \& Carp, D. (2016). Optimization of Container Stowage in a Yard Blocks Using a Genetic Algorithm, Studies in Informatics and Control, 25(1), 123-130. DOI: 10.24846/ v25ily201613

20. Zhang, J., Han, Z., Zhu, F. \& Zhao, X. (2014). Absolute Exponential Stability and Stabilization of Switched Nonlinear Systems, Systems and Control Letters, 66(1), 51-57. 